\title{
RESCUE THERAPY IN MECHANICAL THROMBECTOMY REFRACTORY OCCLUSIONS WITH DETACHABLE STENT-RETRIEVERS AND GP IIB/IIIA INHIBITORS: A SINGLE CENTER EXPERIENCE.
}

\author{
P. Garcia-Bermejo ${ }^{1}$, A. Zakaria², S.N. Patro², N. Akhtar¹, M. Saqqur ${ }^{1}$, S. Kamran¹, G. Al Rumaihi ${ }^{3}$, Y.Z.B.

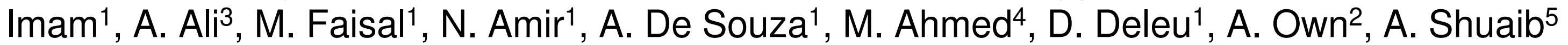

Neurology Department ${ }^{1}$, Neuroradiology Department ${ }^{2}$, Neurosurgery Department ${ }^{3}$, Clinical Pharmacy Department ${ }^{4}$, Neuroscience Institute ${ }^{5}$. Hamad General Hospital. Hamad Medical Corporation. Doha, Qatar.

\section{Background}

Mechanical Thrombectomy (MT) with stent-retrievers is standard of care. Around $25 \%$ of the cases do not reach reperfusion, and Rescue Therapies (RTs) are controversial(1). We aim to present our experience treating refractory occlusions $(\mathrm{RO})$ by means of eptifibatide (GP Ilb/Illa inhibitors) and intracranial stent detachment.

\section{Methods}

We retrospectively evaluated patients treated with MT in our Center in Doha (Qatar), from April 2015 to November 2017. Those with $\mathrm{RO}$ (lack of reperfusion or $\mathrm{mTICl} \leq 2 \mathrm{a}$ ) underwent "off protocol" RT if unstable critical stenosis was detected after 1-2 passes of stent-retriever. Different approaches were chosen based on expert preference. A group of patients was treated with eptifibatide alone, whereas another with intracranial stent detachment plus eptifibatide. Radiological and clinical outcome will be described and compared between both groups

Figures: Case of rescue therapy with eptifibatide IA plus permanent stenting: 36 -year-old Bangladeshi male, with a "wake up" complete left MCA syndrome (NIHSS 20).

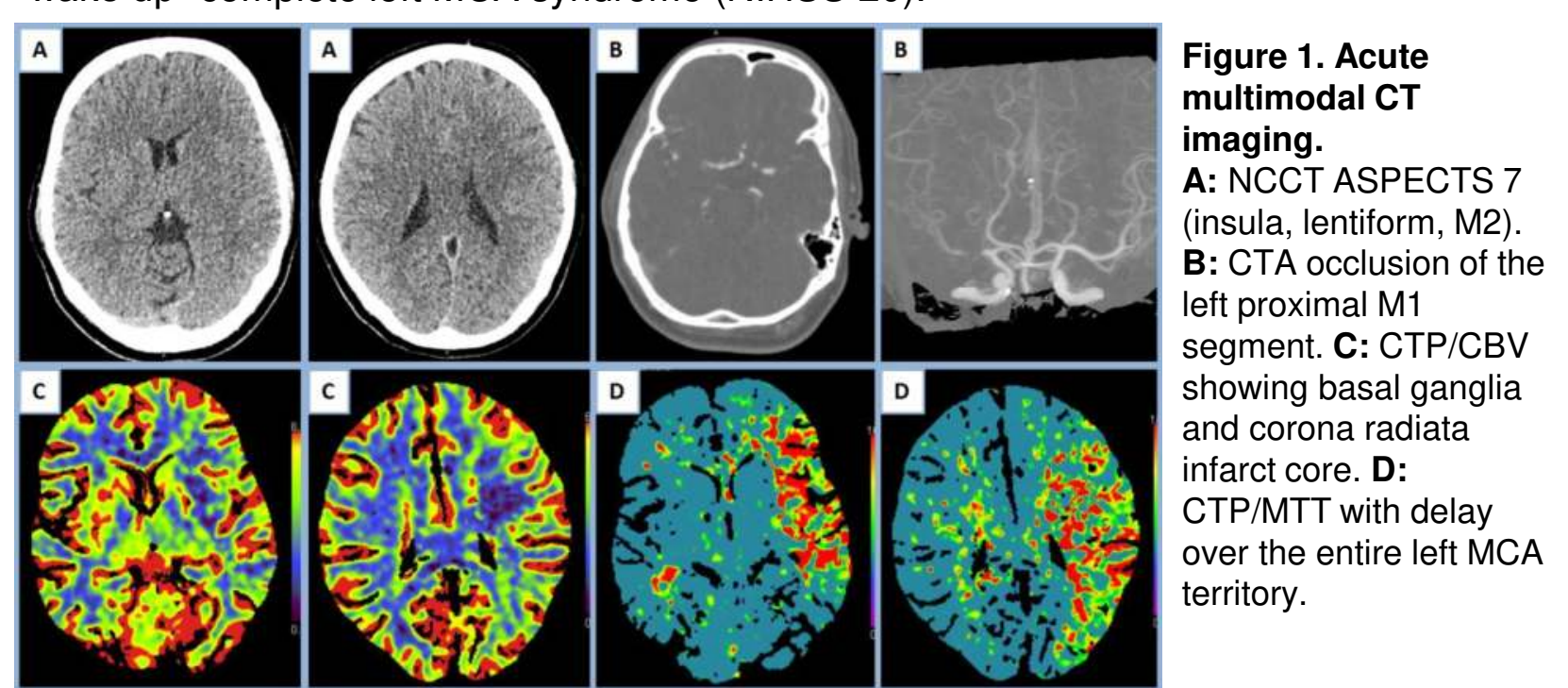

Figure 2. Mechanical thrombectomy. A: left proximal Mocclusion. B: complete recanalization after pass of stentretriever. C: Early spontaneous severe re-stenosis after 10 minutes. D: by pass min. D. by pass of devchabe stentrever over lesion after IA bolus of eptifibatide. E, F anteroposterior and lateral view of complete recanalization after detachment of stent.

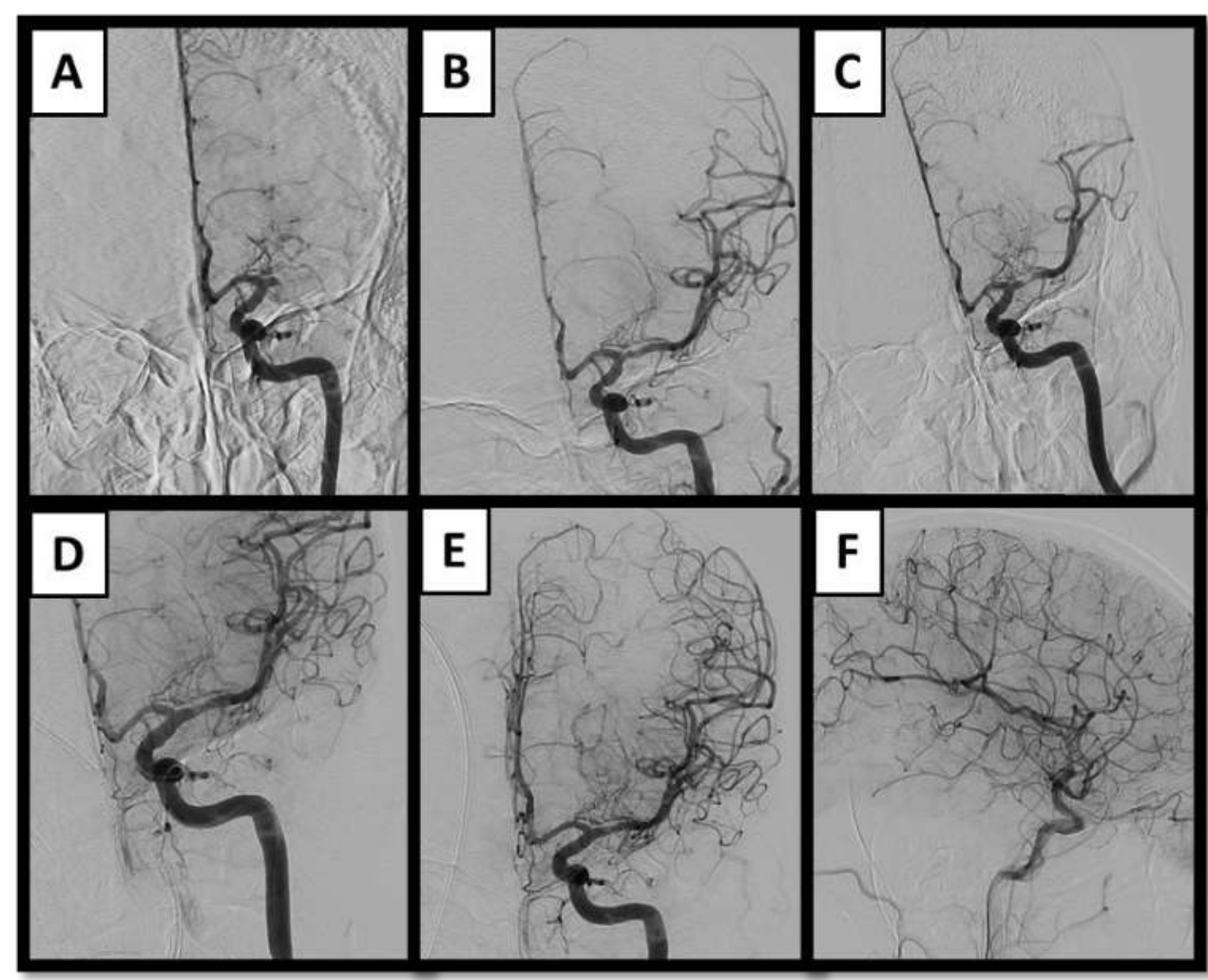

\section{Results}

During the study period, 103 patients were selected for MT. 17 (16.5\%) underwent RT (5 (29.4\%) eftifibatide only and 12 $(70.6 \%)$ stenting plus eptifibatide). 3 (17.6\%) cases did not recanalise (2 from eptifibatide group). 2 experienced reocclusion on follow up vessel imaging despite initial successful recanalization (one from each group). No patient experienced symptomatic hemorrhagic transformation. However 1 patient died due to malignant infarction. 6 (35.29\%) had good outcome (mRS $\leq 2)$ at 90 days (25\% eptifibatide vs $50 \%$ stenting; $p=$ 0.58). (Table 1).

Table 1. Univariate analysis comparison between Rescue Therapy modality groups.

\begin{tabular}{|c|c|c|c|}
\hline Variables & $\begin{array}{c}\text { Eptifibatide only } \\
\qquad(\mathrm{N}=5)\end{array}$ &  & $P$ value \\
\hline Mean Age $\pm S D$, years & $38.4 \pm 6.80$ & $54.5 \pm 13.33$ & 0.005 \\
\hline Male Gender, N (\%) & $5(100)$ & $9(75)$ & 0.51 \\
\hline Median Baseline NIHSS (IQR) & $15(10-21)$ & $11(4.5-16)$ & 0.19 \\
\hline Iv thrombolysis, $\mathrm{N}(\%)$ & $1(20)$ & $4(33.3)$ & 1.00 \\
\hline $\begin{array}{l}\text { Median Baseline CT ASPECTS } \\
\text { (IQR) }\end{array}$ & $8(8-9)$ & $10(9-10)$ & 0.06 \\
\hline Mean Number of passes \pm SD & $3.2 \pm 1.30$ & $2.42 \pm 1.08$ & 0.27 \\
\hline Reperfusion (mTICI 2b-3), N (\%) & $3(60)$ & $11(91.6)$ & 0.17 \\
\hline Median 24h CT ASPECTS (IQR) & $7(5-8.5)$ & $8.5(5.75-9)$ & 0.51 \\
\hline FU MRA, N (\%) & & & 0.004 \\
\hline - Occlusion & $3(60)$ & $1(8.3)$ & \\
\hline - $\quad$ Stenosis $>50 \%$ & 0 & 0 & \\
\hline - $\quad$ Stenosis $<50 \%$ & $2(40)$ & 0 & \\
\hline - Full recanalization & 0 & $8(66.7)$ & \\
\hline \multicolumn{4}{|l|}{$\begin{array}{l}\text { Median Workflow times (IQR), } \\
\text { minutes }\end{array}$} \\
\hline - Door to needle & $30(27-30)$ & $52.5(15-96)$ & 1.00 \\
\hline - Door to CT & $23(15-53)$ & $19(14-28)$ & 0.620 \\
\hline - $\quad$ CT to GP & $83(73-107)$ & $104(54-131)$ & 0.294 \\
\hline - $\quad$ GP to recanalization & $49(37-100)$ & $73(34-91)$ & 1.00 \\
\hline $\mathrm{mRS} \leq 2$ at 90 days, $\mathrm{N}(\%)$ & $1(25)$ & $5(50)$ & 0.58 \\
\hline
\end{tabular}

\section{Conclusion}

Rescue therapy for $\mathrm{RO}$ could be a necessary approach to increase the rate of recanalization and functional independence. We have described our experience with different techniques, which requires further discussion and study.
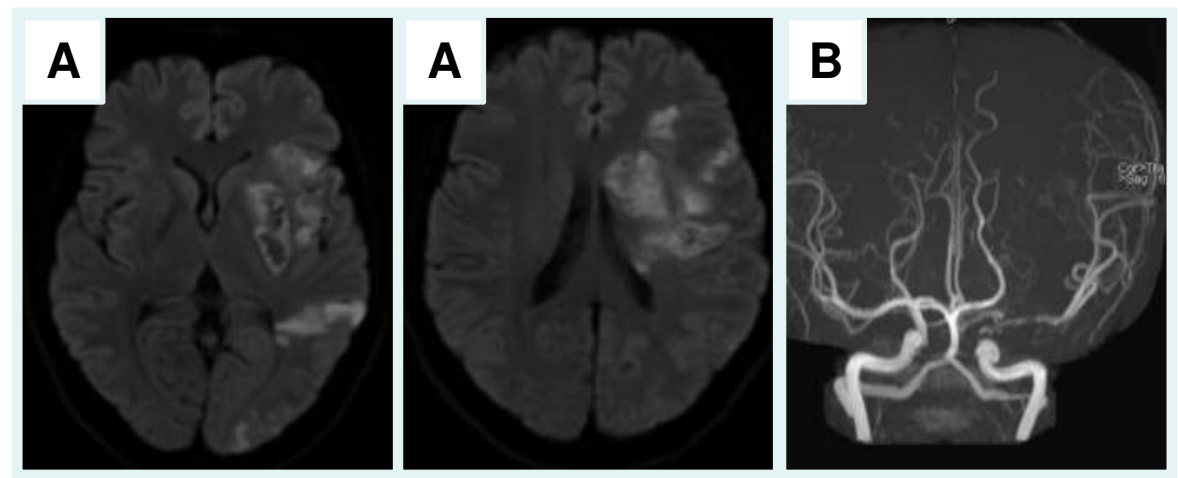

Figure 3. Follow up 24 h MRI. A: DWI showing infarction at the left MCA territory. B: TOF MRA showing artifact at the distal left ICA and M1 segments with distal flow, corresponding with patency of the stent. 